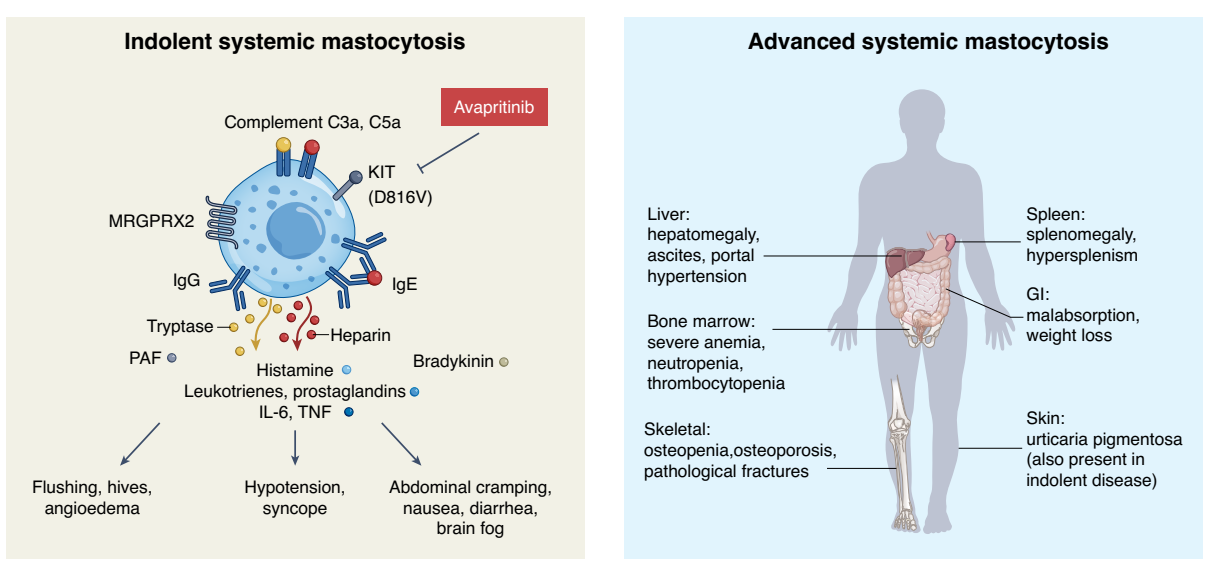

Fig. 1 | SM symptoms, mediators and organ impact of indolent and advanced disease. MRGPRX2, G protein-coupled receptor; IgG and IgE, immunoglobulins; PAF, platelet-activating factor; IL-6, interleukin 6; TNF, tumor-necrosis factor; GI, gastrointestinal.

before this exclusion. Other side effects in PATHFINDER included cognitive adverse events, which occurred in $11 \%$ of patients and were generally mild. Interestingly, the cognitive domain in the EORTC-QLQ-C30 quality-of-life scale improved overall, despite these adverse events; the mechanisms underlying these events are yet to be elucidated.

Advanced SM is a complex and heterogeneous disease often associated with other myeloproliferative neoplasms and other mutations, in addition to the KIT D816V mutation. Both the EXPLORER trial and the PATHFINDER trial excluded patients with acute myeloid leukemia or very-high-risk myelodysplastic syndrome, as defined by the Revised International Prognostic Scoring System. The optimal treatment strategies for advanced SM associated with these poor prognostic factors remain to be determined, and may be further guided by individual mutational profiling.
The use of avapritinib should be guided by a proactive monitoring strategy to identify patients who develop thrombocytopenia and may be at risk for intracranial bleed, which will facilitate appropriate dose modifications or discontinuation of the drug. The effect of avapritinib in terms of symptom reduction and decrease in skin lesions is encouraging not only for patients with advanced SM but also for those with indolent SM, who typically carry only the KIT D816V mutation and may be more amenable to therapies that specifically target this variant $^{12,13}$. Preliminary results from an ongoing study using avapritinib in low doses in patients with indolent SM yielded positive results in terms of both symptom reduction and a decrease in markers of mast-cell burden, with no serious adverse events ${ }^{13}$. Treatment of symptomatic patients with indolent SM concurrently with anti-mediator treatment (e.g., antihistamines or mast-cell stabilizers) may expand the clinical use of these drugs in the future in select populations of patients with indolent disease.

Overall, data from the EXPLORER and PATHFINDER studies strongly support avapritinib as a first-line treatment option in patients with advanced SM, with the exception of those with thrombocytopenia and those with substantial baseline cognitive impairments. Future trials may focus on the role of KIT D816V-selective inhibitors as part of combination regimens with other cytoreductive approaches in patients with high-risk advanced SM.

\section{Mariana Castells $\mathrm{s}^{1,2 \times}$ and Cem Akin ${ }^{3}$ ${ }^{1}$ Division of Allergy and Clinical Immunology, Brigham and Women's Hospital, Boston, MA, USA. ${ }^{2}$ Department of Medicine, Harvard Medical School, Boston, MA, USA. ${ }^{3}$ Division of Allergy and Immunology, Department of Internal Medicine, University of Michigan, Ann Arbor, MI, USA.

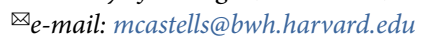

Published online: 6 December 2021 https://doi.org/10.1038/s41591-021-01588-z

References

1. Valent, P., Akin, C. \& Metcalfe, D. D. Blood 129, 1420-1427 (2017).

2. Metcalfe, D. D. J. Invest. Dermatol. 96, 2S-4S (1991).

3. Castells, M. \& Austen, K. F. Int. Arch. Allergy Immunol. 127, 147-152 (2002)

4. Theoharides, T. C., Valent, P. \& Akin, C. N. Engl. J. Med. 373, 163-172 (2015)

5. Escribano, L., Akin, C., Castells, M., Orfao, A. \& Metcalfe, D. D. Ann. Hematol. 81, 677-690 (2002).

6. Escribano, L. et al. J. Allergy Clin. Immunol. 124, 514-521 (2009).

7. Lim, K. H. et al. Blood 113, 5727-5736 (2009).

8. Nagata, H. et al. Proc. Natl Acad. Sci. USA 92, 10560-10564 (1995)

9. DeAngelo, D. J. et al. Nat. Med. https://doi.org/10.1038/s41591 021-01538-9 (2021)

10. Gotlib, J. et al. Nat. Med. https://doi.org/10.1038/s41591-02101539-8 (2021).

11. Gotlib, J. et al. N. Engl. J. Med. 374, 2530-2541 (2016).

12. Castells, M. \& Butterfield, J. J. Allergy Clin. Immunol. Pract. 7, 1097-1106 (2019).

13. Akin et al. J. Allergy Clin. Immunol. 145, AB336 (2020).

\section{Competing interests}

M.C. and C.A. have consultancy agreement with and receive research funding from Blueprint Medicines.

\title{
mRNA vaccines offer hope for HIV
}

mRNA technology may be uniquely positioned to tackle a major hurdle for HIV vaccines: the elicitation of broadly cross-reactive neutralizing antibodies. A preclinical study takes the first step toward this goal.

\section{Lynn Morris}

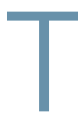

he remarkable success of mRNA vaccines against COVID-19 has been nothing short of miraculous.
Whether this unique technology platform can be used to tackle the more complex task of developing a vaccine against human immunodeficiency virus (HIV) is now under intense scrutiny. In this issue of Nature Medicine, a preclinical study by Zhang et al. 

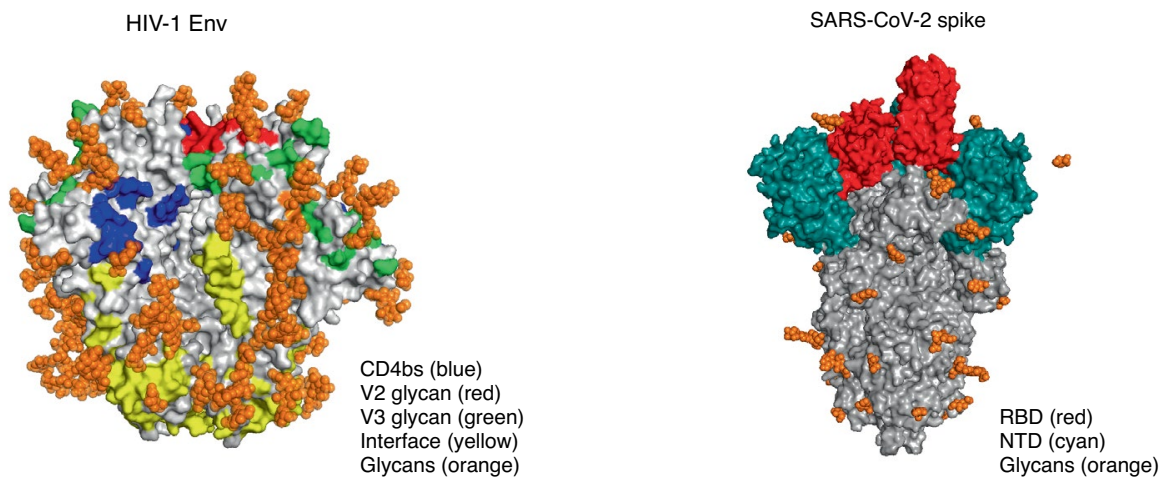

Fig. 1 | Complexity of HIV-1 Env versus SARS-CoV-2 spike. HIV Env (left) has multiple conformational bNAb epitopes (four of the six epitopes are shown here), is densely covered in glycans, and elicits a polyclonal neutralizing antibody response. Genes encoding antibodies to Env are heavily mutated, and bNAbs are rarely elicited by infection or vaccination. In contrast, the SARS-CoV-2 spike protein (right) is lightly covered in glycans, and the receptor-binding domain (RBD) is the main immunodominant epitope, with the $\mathrm{N}$-terminal domain (NTD) targeted to a lesser extent. It generates a focused neutralizing antibody response; genes encoding antibodies to spike protein have limited or no mutations, and bNAbs are commonly elicited after infection and vaccination. CD4bs, CD4-binding site. Images from RCSB Protein Data Bank (https://www.rcsb.org/) accession codes 4ZMJ (glycans transposed from 5FUU; HIV Env) and 6VSB (glycans transposed from 7CN9; SARS-CoV-2 spike protein). We thank T. Moyo-Gwete for preparation of this figure.

suggests that the mRNA platform may be up to the challenge ${ }^{1}$. The authors encapsulated mRNA encoding the HIV envelope glycoprotein (Env) (the equivalent of the SARS-CoV-2 spike protein), together with the structural HIV group-specific antigen protein (Gag), in a lipid nanoparticle, to produce virus-like particles (VLPs) in vivo. These Env-expressing VLPs elicited broadly neutralizing antibodies (bNAbs) and other immune responses that were protective against viral challenge in a macaque model. Although the VLPs were not nearly as immunogenic or as efficacious as mRNA vaccines against COVID-19, these results are encouraging and illuminate a pathway toward inducing the higher and more-durable antibody responses needed to prevent infection with HIV.

This is no small feat; Env is a formidable target that fails to induce the right kind of antibodies even in the majority of people who are infected with HIV. Unlike spike protein, which is relatively stable and contains just a few immunodominant epitopes (mostly restricted to the receptor-binding domain), Env is a complex trimeric protein with multiple dispersed antibody epitopes - many of which are conformational and heavily coated in sugars that shield them from antibody attack (Fig. 1). The ability to generate soluble trimeric Env proteins through the introduction of key mutations was a major advance in immunogen design, although these so-called
'SOSIP proteins' induced only autologous strain-specific neutralizing antibodies ${ }^{2}$. In the present study by Zhang et al., antibodies elicited by the Env-Gag mRNA were able to neutralize almost all members of a global panel of HIV isolates, classified as having a tier 2 phenotype ${ }^{1}$. This represents a major step forward for a vaccine against HIV, as this phenotype is typical of most circulating strains, which are difficult to neutralize due to a closed Env conformation.

It is likely that a number of factors contributed to the greater immunogenicity of the mRNA vaccine designed by Zhang et al. ${ }^{1}$. The endogenous expression of the native Env on the surface of a VLP would preserve conformational epitopes, and VLPs were also shown to contain double the number of Env molecules present in an HIV viral particle. Furthermore, mRNA continues to be expressed for several days after administration, providing ongoing immunostimulation $^{3}$. Another potential advantage is the ability of VLPs to bind to antigen-presenting cells, which guarantees delivery of mRNA into the appropriate cells and the induction of follicular helper $\mathrm{T}$ cells that are crucial for B cell development in germinal centers ${ }^{4}$. Zhang et al. also chose an HIV Env sequence that lacks a glycan at position 276; this enabled better access to the B cell precursors of bNAbs that target the CD4-binding site on the viral envelope ${ }^{1}$. Indeed, Env-Gag mRNA elicited antibodies to that site and, interestingly, to many other HIV epitopes - a considerable improvement on the strain-specific 'glycan-hole' responses seen with SOSIP proteins. It will be important in future studies to isolate B cells from mRNA-immunized animals to ascertain whether they are capable of broad neutralization and if they carry the genetic features associated with known bNAb lineages. Although the results from Zhang et al. ${ }^{1}$ are encouraging for the HIV vaccine field, this is a complex and impractical protocol that requires multiple immunizations with high doses of mRNA. Moreover, the levels of bNAbs elicited in this study were extremely low and took a year to develop, and their role in protection from infection remains unclear.

mRNA technology has only recently come of age; it was originally hampered by instability and unfavorable immunogenicity, but decades of research have solved these problems, and the advantages of mRNA as a vaccine platform continue to emerge ${ }^{5}$. These include rapid development, ease of manufacture and scalability, which offer advantages over the traditional vector-based or protein-based vaccines being pursued in the HIV field. For example, mRNA would enable testing of sequential immunization and lineage-based approaches that require multiple immunogens with minor but important sequence changes ${ }^{6} . \mathrm{mRNA}$ is also considerably cheaper to produce and can be modified as needed, an important consideration for rapidly mutating viruses like HIV. Among the vaccines against COVID-19, those based on the mRNA platform have superior immunogenicity and stimulate both B cell responses and $\mathrm{T}$ cell responses ${ }^{7,8}$. The precise mechanisms underlying mRNA immunogenicity, however, are still unknown, and much remains to be learned if they are to be optimized and applied to HIV prevention. Nonetheless, the vast amount of safety data available from mRNA vaccines against COVID-19 will probably contribute to streamlined regulatory approval processes for vaccines against HIV and other diseases.

In contrast to the swift success of vaccines against COVID-19, the story of the development of vaccines against HIV has been long and troubled. Of the eight vaccine efficacy trials conducted so far, only one (with a viral-vector and protein-based vaccine prime-boost regimen) has shown moderate efficacy, although the follow-up trial failed to replicate this result ${ }^{9}$. This has shifted the field back to the pursuit of a vaccine able to induce bNAbs. Recently, the Antibody-Mediated Prevention clinical trials demonstrated that a monoclonal bNAb against the CD4-binding site can prevent infection of humans with 
$\mathrm{HIV}^{10}$, although it came with the sobering realization that high levels of bNAbs will probably be required (P. Gilbert, personal communication). Another approach to generating bNAbs is to trigger the $\mathrm{B}$ cell precursors of specific bNAb lineages. One such germline-targeting immunogen is eOD-GT8, a nanoparticle coated with HIV Env gp120 proteins, which binds rare B cells specific for the CD4-binding site in monkeys and humans ${ }^{11}$. eOD-GT8 has now been converted into an mRNA vaccine through the same platform as the successful Moderna vaccine against COVID-19, with human clinical trials due to start soon.

There is no doubt that HIV presents a much greater challenge for vaccine developers than COVID-19 does. The vast genetic diversity and the ability of
HIV to integrate into the human genome necessities that a vaccine elicits antibodies able to block every viral particle. Whether further optimization of Env immunogens, together with the strong priming effect of an mRNA vaccine platform, is able to achieve this will require further investigation. The hope is that the lessons learned from the development of vaccines against COVID-19 will be used to solve the HIV problem and that this will be tackled with the same sense of urgency, given that HIV remains a major global health challenge.

\section{Lynn Morris (D) 1,2区}

${ }^{1}$ Antibody Immunity Research Unit, Faculty of Health Sciences, University of the Witwatersrand, Johannesburg, South Africa. ${ }^{2}$ Center for the AIDS Program of Research in South Africa, University of
KwaZulu-Natal, Durban, South Africa.

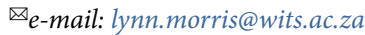

Published online: 9 December 2021

https://doi.org/10.1038/s41591-021-01602-4

References

1. Zhang. P. et al. Nat. Med. https://doi.org/10.1038/s41591-02101574-5 (2021).

2. Sanders, R. W. et al. Science 349, aac4223 (2015).

3. Pardi, N. et al. J. Control. Release 217, 345-351 (2015).

4. Pardi, N. et al. J. Exp. Med. 215, 1571-1588 (2018).

5. Chaudhary, N., Weissman, D. \& Whitehead, K. A. Nat. Rev. Drug Discov. 20, 817-838 (2021).

6. Mu, Z., Haynes, B. F. \& Cain, D. W. Vaccines 9, 134 (2021).

7. Khoury, D. S. et al. Nat. Med. 27, 1205-1211 (2021).

8. Lederer, K. et al. Immunity 53, 1281-1295.e5 (2020).

9. Gray, G. E. et al. N. Engl. J. Med. 384, 1089-1100 (2021).

10. Corey, L. et al. N. Engl. J. Med. 384, 1003-1014 (2021).

11. Jardine, J. G. et al. Science 351, 1458-1463 (2016).

Competing interests

The author declares no competing interests. 